\title{
Proceedings of the 129th Semon Club, 27 May 2005, the Otolaryngology Department, Guy's and St Thomas' NHS Foundation Trust, London, UK
}

Chairman: Miss E Chevretton, Consultant ENT Surgeon, Guy's and St Thomas' NHS Foundation Trust, London

Pathologists: Professor L Michaels, University College London, and Dr I Clarke, University College London and The Royal Free Hospital, London

Radiologist: Ms R Preston, Specialist Registrar, Guy's and St Thomas' NHS Foundation Trust, London

Secretary: Mr D Gillett, Specialist Registrar, Guy's and St Thomas' NHS Foundation Trust, London

\section{Oncology and skull base session}

Chairman: Mr G Morrison

\section{Pigmented villonodular synovitis of the temporomandibular joint, presenting as a pre-auricular mass}

D M Wolf, T Odutoye, A Deery

From St George's Hospital, London, UK

We presented the case of a 43-year-old Caucasian man who presented with a three-week history of a left-sided pre-auricular mass. A magnetic resonance imaging scan revealed a dumbbell-shaped lesion arising from the left temporomandibular joint synovium. Fine needle aspiration cytology and further immunocytochemistry suggested the diagnosis of pigmented villonodular synovitis of the temporomandibular joint. Giant cell tumours of the tendon sheath are the second most common tumours of the hand. These soft tissue tumours have no gender predilection, and it is the diffuse type (also known as pigmented villonodular synovitis) that occurs more commonly in larger joints, including the temporomandibular joint. Presentation is usually with a painless swelling of insidious onset and may include trismus, clicking and pain. Treatment consists of wide local excision including involved bone and synovium, but recurrence rates may be as high as 46 per cent

In discussion, Professor Michaels agreed with the cytological findings and, combining this information with the clinical and radiological features, was confident of the diagnosis.

The aim in presenting this case was to increase awareness of temporomandibular disease. Although rare, it is important to include pigmented villonodular synovitis in the differential diagnosis of pre-auricular swellings.

\section{An unusual cause for a conductive hearing loss \\ R Ganesalingham, I Friedrichs, J Knight From St George's Hospital, London, UK}

A 35-year-old woman presented with intermitted infection and increasing hearing loss in her right ear. A pure tone audiogram showed a severe conductive hearing loss in that ear. She underwent a diagnostic tympanotomy which revealed a cystic mass filling the middle-ear cavity, extending into the mastoid. A subsequent computed tomography scan confirmed the findings. The ossicles were intact and there was no evidence of any bony erosion. The lesion was removed and histological analysis revealed a lipoma.

We presented this case at the Semon Club because of the unusual diagnosis of a lipoma of the middle ear and in order to discuss further management of the patient. There are only five previous cases in the literature: four presented with eustachian tube dysfunction and one with an upper airway obstruction. Professor Michaels, however, doubted the diagnosis of middle-ear lipoma. He indicated that the slide contained middle-ear mucosa, muscle and fat cells, which would be consistent with a biopsy of the tensor tympani muscle. The slide also represented a rather small sample of the rather large middle-ear lesion, and the true pathology might have been missed. Ms Preston added that the radiological features were more consistent with those of a cholesterol granuloma.

\section{Small cell neuroendocrine tumour of the parotid gland}

D Bray, C Dutta, A Owa

From the Harold Wood Hospital, Romford, UK

We discussed the presentation, diagnosis, pathological features, management strategies and prognosis of neuroendocrine carcinoma of the parotid gland. A 77-year-old man presented with a non-tender lump in the left side of the neck. Examination revealed a $2 \times 2 \mathrm{~cm}$, oval, fixed mass by the left angle of the mandible. Cytology showed reactive lymphadenopathy. A computed tomography scan demonstrated a large, $3 \mathrm{~cm}$ mass in the left parotid region. The patient underwent left superficial parotidectomy. Radiotherapy to the left parotid and neck region was given.

Neuroendocrine neoplasms have either epithelial or neural origins and originate from the neurons or the paraganglia of the peripheral nervous system. They range from atypical or well differentiated to high-grade neoplasms. Small cell carcinomas in the salivary glands are extremely rare, accounting for less than 1 per cent of all parotid gland carcinomas and 3.5 per cent of all malignant salivary gland tumours. In the head and neck, small cell carcinomas have been reported in the larynx, pharynx, paranasal sinuses, trachea and oral cavity.

The radiological features were consistent with a tumour although no distinguishing features were detected. The role of positron emission tomography scanning was highlighted and this investigation recommended. The histology was discussed and the beneficial roles of frozen section and neck dissection in these aggressive tumours debated.

In conclusion, these rare tumours are potentially curable and should be treated as such.

Complications of routine middle-ear surgery, secondary to previous radiotherapy

I Friedrichs, D Wolf, I J Knight

From St George's Hospital, London, UK

A 58-year-old man had 11 years prior been successfully treated with radiotherapy for a non-Hodgkin lymphoma 
in his postnasal space. In December 2003, he presented with a conductive hearing loss in his right ear and was diagnosed as suffering from otosclerosis in that ear. In May 2004, he underwent stapedectomy in that ear. After the surgery, he continued to suffer from intractable otalgia and discharge in the operated ear. He was treated with numerous topical antibiotics but his symptoms persisted. With the progression of his symptoms, he developed pale granulation tissue in his ear canal, which we biopsied in December 2004 but which was reported as inflammatory tissue only. He also underwent a computed tomography scan of his temporal bone in December 2004. The scan showed abnormal soft tissue in the whole middle ear and bony destruction of the ear canal. A white blood cell labelled nuclear medicine scan of the patient's temporal bone confirmed skull base osteomyelitis. He was subsequently admitted for triple antibiotic treatment with ciprofloxacillin, metronidazole and ceftriaxone and topical treatment with gentamycin. However, his symptoms did not improve over his six-week hospital stay and he was discharged again. At the time of writing, the patient was still suffering from otalgia and discharge in his operated ear. He had not developed any cranial nerve palsies thus far.

\section{Ectopic meningioma: a case report with literature review}

G Kumar, S Basu, P Sen, S A Kamal

From the Whipps Cross University Hospital, London, UK

Meningiomas are the commonest benign intracranial tumour, accounting for 13-18 per cent of all primary intracranial tumours. They are generally slow-growing tumours composed of neoplastic arachnoidal cells. Ectopic (extradural) meningiomas that do not originate from either the optic fascicle or the intracranial meninges are exceedingly rare.

A patient with an ectopic meningioma in the external auditory canal and mastoid, without intracranial extension, was treated in our unit. This 45 -year-old man presented with left conductive deafness and left ear discharge. On examination, there was sagging of the left posterior canal wall, and a left modified radical mastoidectomy was performed. Histology was suggestive of meningioma. A postoperative follow-up magnetic resonance imaging scan of the brain and temporal bone ruled out intracranial extension.

In discussion, Professor Michaels expressed the opinion that a temporal bone meningioma diagnosis is made by histology and immunohistochemistry and that an intracranial component must be ruled out.

Surgical excision of this condition is the mainstay of treatment and often requires the involvement of a team consisting of an otolaryngologist, head and neck surgeon, and neurosurgeon. The otolaryngologist should be well aware of the need for long-term follow up of this group of patients, with regular scanning to rule out any intracranial foci or recurrence of the tumour.

\section{Congenital semicircular canal dehiscence}

N Donnelly, A Fitzgerald O'Connor

From the Guy's \& St Thomas' NHS Foundation Trust, London, UK

We presented the case of a 64-year-old lady with a 30-year history of hearing loss following head trauma. Associated symptoms included pulsatile tinnitus, disequilibrium and oscillopsia. She had no history of sound- or pressure-induced vertigo, but there was a family history of hearing and balance disturbance.
Examination revealed normal tympanic membranes, with some tympanosclerosis, and the patient was Rinnenegative. Clinical vestibular assessment was normal. Audiometry displayed a symmetrical, mixed, predominantly conductive hearing loss. Stapedial reflexes were absent. A dehiscence in each superior semicircular canal was seen using high resolution computed tomography (CT) scanning, with no features of otosclerosis.

Superior semicircular canal dehiscence syndrome has been recently reported. It is typically unilateral and associated with sound- or pressure-induced vertigo. Audiological features include normal to severe mixed hearing loss, with normal stapedial reflexes.

Discussion confirmed that the radiological criteria for the diagnosis of canal dehiscence had been met. In the absence of other aetiologies, the canal dehiscence was considered the cause for the symptoms, despite atypical features.

In conclusion, high-resolution CT images of the temporal bone are required before diagnosing otosclerosis and proceeding to surgery.

\section{Upper airways obstruction session}

Chairman: Mr S Blaney

\section{Intra-laryngeal thyroid tissue presenting with upper airway obstruction}

P Ajulo, C Skilbeck*, A Jacob*

From Guy's Hospital and the *University Hospital Lewisham, London, UK

Ectopic thyroid tissue in the lateral neck and along the thyroglossal tract is not uncommon; intralaryngeal ectopic thyroid tissue is extremely rare.

A 62-year-old African woman presented with hoarseness, dyspnoea and dysphagia 13 years after surgery for benign thyroid enlargement. Fibre-optic laryngoscopy revealed a large, partly cystic right supraglottic mass. Computed tomography of the neck showed a large, heterogeneous mass grossly distorting the airway, with a stalk connecting it to the right hemithyroid remnant in the anterior neck but no bony or cartilaginous erosion. Microlaryngoscopy and biopsy were performed with a covering tracheostomy due to bleeding from this highly vascular lesion. Histology showed the mass to be benign thyroid tissue, subsequently debulked with carbon dioxide laser. The patient was successfully decannulated two weeks later and discharged home.

We proposed the origin of the laryngeal ectopic thyroid tissue to be growth and herniation of the thyroid remnant through a defect in the deep cervical fascia and thyrohyoid membrane, due to the previous thyroid surgery. We believe that this is the first case reported in the literature.

Definitive surgery, if appropriate, should be via a laryngofissure. Thyrotropin suppression with thyroxine was another option for this patient, given her age, to discourage re-growth.

\section{Lymphoma presenting with stridor, requiring crash tracheostomy}

D Vahidova, H Chau, G Kenyon

From the Whipps Cross University Hospital, London, UK

We presented the case of a 48-year-old man who presented to our accident and emergency department with a fourweek history of increasing breathing difficulty and stridor. He did not respond to conservative treatment and his condition rapidly deteriorated within an hour of presentation. Flexible nasendoscopy, performed in the operating theatre, 
showed a swelling of the posterior pharyngeal wall compromising the upper airway. While in theatre, the patient became completely obstructed. Attempted intubation was unsuccessful, requiring crash tracheostomy to be performed.

Investigations at the time of presentation showed electrolytes and full blood count to be normal except for a mildly low lymphocyte count of 1.2 (normal range 1.5-4). Subsequent computed tomography (CT) scanning showed a $4 \times 6 \mathrm{~cm}$ collection in the prevertebral region, consistent with an abscess starting at the level of $\mathrm{C} 1$ and extending to the level of the vocal folds, narrowing the airway.

Following these CT findings, the patient was taken to theatre for examination and drainage of the abscess. However, no collection was found and a biopsy was taken of the solid mass, which was reported as high-grade B-cell non-Hodgkin's lymphoma.

This is an interesting and rare presentation of lymphoma, which should be considered in the differential diagnosis of future cases even if imaging suggests an abscess.

Discussion ensued following our presentation. We were asked why panendoscopy and biopsy were not performed immediately after the crash tracheostomy, to which we replied that endoscopy had been performed but we were unable to clearly determine the exact cause of airway obstruction at that time. We were also asked why the patient's condition did not improve despite intravenous steroids (lymphomas usually respond well to such treatment), to which we replied that the patient deteriorated rapidly after presentation, without sufficient time for the steroids to have a significant effect.

\section{Ortner's syndrome}

N I K Ibery, P M Patel, P J Hadfield

From the Royal Surrey County Hospital, Guildford, UK

We described the first reported case of Ortner's syndrome following an endovascular cardiac procedure. Ortner's syndrome, or 'cardio-vocal syndrome' is a rare but recognized cause of vocal fold paralysis caused by intra-thoracic pathology. Unless recognized and treated promptly it can be fatal. To date, there have been no published cases of the syndrome following coronary intervention (and possibly being a direct consequence of it).

A 79-year-old gentleman presented to the accident and emergency department with a four-week history of worsening hoarseness, spasmodic cough and occasional chest pain. Six weeks prior to presentation, he had been diagnosed with an acute myocardial infarction and treated with emergency angioplasty and stenting.

On this admission, the chest pain was diagnosed as angina due to his ischaemic heart disease. On ENT examination, he was found to have a fixed, abducted left vocal fold. A computed tomography (CT) scan revealed a large thoracic aortic aneurysm stretching the left recurrent laryngeal nerve. The aneurysm was saccular, measuring $6 \mathrm{~cm}$ in diameter, and was confined to the inferior surface of the aortic arch.

The aneurysm was successfully treated by endovascular stenting and the patient made a full recovery.

In discussion, it was pointed out that the best way to investigate the patient with suspected left recurrent laryngeal nerve palsy would be with the combination of CT of the thorax and magnetic resonance imaging of the head and neck.

This case illustrates that fact that certain common ENT symptoms may be attributable to rare but serious intra-thoracic pathology. A diagnosis of Ortner's syndrome may be delayed or missed. Our aim in presenting this case was to increase clinicians' awareness of these important symptoms to ensure prompt management of this rare but life-threatening diagnosis.

\section{Laryngeal tuberculosis: the masquerading disease}

H Y Ching, A Hope, T Odutoye

From St George's Hospital, London, UK

We report the case of a 42-year-old Caucasian male nurse who presented with a history of dysphagia, odynophagia, dysphonia and dry cough. The clinical features were highly suggestive of laryngeal carcinoma. Out-patient endoscopic examination revealed a completely destroyed epiglottis with ulceration at the base of the tongue and supraglottis. With a working diagnosis of supraglottic carcinoma, a staging computed tomography (CT) scan and formal staging endoscopy were arranged urgently. Surprisingly, the CT scan revealed cavitating chest lesions and other features that were highly suggestive of Mycobacterium tuberculosis (TB) infection. Histopathological investigations revealed widespread epithelioid granulomas without dysplasia or malignancy. The diagnosis was confirmed as laryngeal TB.

With the availability of effective anti-tuberculous drugs in the developed countries, the incidence of laryngeal TB is rare. However, with the rising incidence of acquired immunodeficiency syndrome and the ease of world travel, more cases of the laryngeal presentation of TB may be encountered in this country. This unusual case highlights the importance of considering laryngeal TB in patients suspected of laryngeal carcinoma before embarking on surgical management. A high level of suspicion is vital in order to reduce the risk of transmission to health care providers.

\section{TB or not TB?}

A Michael, Z Shabbir, R Michael, H Kaddour From the Harold Wood Hospital, Romford, UK

A 55-year-old Mauritian staff nurse presented to our clinic with a right neck lump, general malaise, weight loss, night sweats and fever of two months' duration. He was heavy smoker, heavy drinker and was hypertensive. An ENT and head and neck examination revealed a large, diffuse, cystic swelling deep to the right sternomastoid, about $6 \times 6 \mathrm{~cm}$ in diameter. There was no sign of inflammation and the cyst was not attached to the skin. A flexible rhinolaryngoscopy showed displacement of the hypopharynx to the left; however, the patient's vocal folds were normal and freely mobile. Fine needle aspiration (FNA) of the swelling produced $15 \mathrm{ml}$ of greenish-yellow, thick pus that was acid-fast bacilli (AFB) positive; however, sputum was AFB-negative. Other laboratory investigations were normal. A barium swallow showed extrinsic swelling pushing the hypopharynx to the left. A magnetic resonance imaging scan showed a multilocular parapharyngeal abscess with a wedged, compressed C4/5. Our management plan comprised repeated ultrasound (US)-guided fine needle aspiration (of about $160 \mathrm{mls}$ of pus each time), commencement on triple antituberculous chemotherapy and neurosurgical referral for further management of the patient's cervical TB osteitis.

Tuberculous deep neck abscesses are relatively rare in the Western World. However, there has been a recent increase in the incidence of TB infection due to many factors, such as breakdown in health services and the increased number of human immunodeficiency virus patients. We recommend that such cases be managed with anti-TB chemotherapy as well as repeated US-guided FNA. This approach has many advantages over traditional surgical drainage: it is a procedure which is office-based, 
relatively safe, repeatable, efficient and, above all, cost-effective.

\section{Head and neck session}

Chairman: Mr S Blaney

\section{A painful clinical dilemma}

C Neumann, K Desai, R Tranter

From the Brighton and Sussex University Hospitals Trust, Brighton, UK

A 72-year-old man diagnosed eight years prior with chronic lymphoid leukaemia (CLL) with splenic involvement presented five years ago with an ulcerative lesion on the right conchal bowl. He underwent no less than five excision biopsies and two excision and grafting procedures, all of which failed to control the local disease and had little effect on the pain, which was the main symptom experienced.

The histological diagnosis was initial basal cell carcinoma followed by solar keratosis and Bowen's disease. An inflammatory element involving the cartilage had been present throughout. Abnormal lymphocytes were not detected in the pinna biopsy, even though lymphoma/ leukaemia has been reported to present as perichondritis. The exacerbation in symptoms coincided with worsening of the patient's general condition in the context of the CLL, and he finally underwent a splenectomy for anaemia. Symptoms were partially controlled with topical therapy using steroids, antifungals and antibiotics combined with oral ciprofloxacin at times.

In discussion, excision of the ulcerated lesion and underlying cartilage followed by flap reconstruction of the pinna and referral to a pain clinic were the options suggested by the panel. Even though the patient entertained the possibility of extirpation of the ear in an attempt to control the pain, this was unlikely to benefit him as the pain had a neuropathic character.

\section{Ancient schwannomal synovial sarcoma: a midline neck lump}

D E J Whitehead, S Gane, P O'Flynn

From the Royal National Throat, Nose and Ear Hospital, London, UK

A 47-year-old man was referred to his local ENT department with a four-week history of a left-sided neck mass. The lump had initially been small and symptom-free but had enlarged and become uncomfortable. The $3 \mathrm{~cm}$ mass to the left of the cricoid cartilage clearly moved on swallowing. An ultrasound arranged by the patient's general practitioner showed a large, heterogeneous, vascular lump in the left anterior neck that was not continuous with either the thyroid or submandibular glands. No lymphadenopathy was noted. Examination was unremarkable. A computed tomography scan and fine needle aspiration cytology were performed. The initial cytology report suggested a malignant mesenchymal lesion, and a second opinion was sought due to the lesion's unusual appearance. It was advised that the lesion warranted urgent removal. The cytology report stated the differential diagnosis as including 'ancient schwannomal synovial sarcoma'. Despite being advised to have the mass removed, the patient declined an operation.

The patient returned three years later with a more midline mass the size and shape of a tennis ball. Prompt excision of this mass was arranged. The firm, spherical mass was encased in dense fibrous tissue and was strongly adherent to the left-sided strap muscles. Histology showed a $5 \mathrm{~cm}$, high-grade sarcoma.

In discussion, Dr Sandison reviewed the histology and commented on the unusual staining for only CD34 but agreed with the diagnosis of sarcoma. It was remarked that, although the lesion was fully excised, the excision margins should have probably included the strap muscles.

\section{Primary malignant supraglottic laryngo-cutaneous fistula}

J Hughes, J Panesar, M Keene

From St Bartholomew's Hospital, London, UK

A 69-year-old male smoker presented with a nine-month history of dysphonia. Examination revealed a $1 \times 1 \mathrm{~cm}$, ulcerated mass involving the posterior surface of the epiglottis. Biopsy suggested cellular atypia but no malignancy. Computed tomography (CT) and magnetic resonance imaging demonstrated nodularity of the epiglottis but no destructive lesion. Ten subsequent biopsies were reported with differing histological findings, including mild to moderate dysplasia, chronic inflammation and papillomatosis appearances. Medical opinions excluded tuberculosis, syphilis and connective tissue disorders.

The patient's condition progressed, with worsening stridor, and laser debulking procedures were undertaken. A mass involving the left thyroid cartilage developed, and fine needle aspiration cytology showed normal chondroid matrix. The mass suppurated and surgical exploration revealed a cutaneous fistula. A further biopsy revealed extensive, severe dysplasia, with areas of invasive squamous cell carcinoma. A CT scan revealed erosion of thyroid cartilage, with normal cervical lymph nodes. The patient underwent total laryngectomy almost five years after his initial presentation and received chemoradiotherapy.

In discussion, Mr Simo acknowledged the difficulty in obtaining diagnostic samples in some cases of laryngeal carcinoma and the need for repeated and deep biopsies. The panel members were not aware of other cases of primary, malignant, supraglottic laryngo-cutaneous fistula; a review of the literature failed to demonstrate a previous report.

\section{An atypical midline cervical neck swelling}

N L Jacobsen, A Jacob, A D'Souza

From the University Hospital Lewisham, London, UK

A 42-year-old lady, with a six-year history of Hashimoto's thyroiditis controlled on thyroxine, presented with a threeweek history of a midline cervical neck swelling. Thyroid function tests were normal. Examination revealed a well defined nodule in the thyroid isthmus measuring $9 \times 5 \mathrm{~mm}$, confirmed with an ultrasound scan. A fine needle aspirate showed tissue consistent with a branchial cleft cyst. Surgery was performed, excising the thyroid isthmus and the enclosed cyst. The patient was asymptomatic at three-month follow-up. Histopathology showed a cyst lined with respiratory epithelium, with areas of squamous metaplasia. These findings were consistent with a branchial cleft cyst. Sub-epithelial stroma contained prominent lymphoid infiltrate with reactive germinal centres.

Branchial cleft cysts are typically characterized by a lateral neck swelling. Our case had the typical histological features of a branchial cleft cyst, but its midline location did not support this diagnosis. Professor Michaels confirmed the histological features that made a diagnosis of branchial cleft cyst likely. He wondered whether this cyst might have been bronchogenic in origin; however, hyaline cartilage, smooth muscle and bronchial glands were not found 
within its wall, nor a lymph node with sequestered respiratory epithelium. Ultimately, this was a benign developmental cyst, with branchial cyst being the most likely diagnosis.

\section{Rhinology session}

Chairman: Mr S Blaney

\section{A self-resolution of advanced nasal granulomatous disease}

\section{A D Morley, D B Mitchell}

From the Kent and Canterbury Hospital, Canterbury, UK

A 32-year-old lady presented with subacute complete nasal obstruction, periorbital discomfort and odontalgia; there was no other history of note. An initial ENT examination revealed a very worrying nasal septal expansion, with inflamed anterior but normal posterior nasal findings. Sinus X-rays showed a right maxillary sinus fluid level, and paranasal sinus computed tomography showed marked pansinusitis. The patient had a normal full blood count and film, but an erythrocyte sedimentation rate of $72 \mathrm{~mm} / \mathrm{hr}$, a C-reactive protein concentration of $58.8 \mathrm{mg} / \mathrm{l}$ and raised concentrations of liver enzymes. Analysis for anti-neutrophilic cytoplasmic antibodies was negative and the angiotensin-converting enzyme concentration was normal but the antithyroglobulin concentration was raised. Multiple mucosal biopsies revealed a marked chronic granulomatous inflammation with patchy vasculitis. Whilst awaiting a definitive multidisciplinary diagnosis, within a few months, the patient recovered fully from her symptoms; examination showed a complete resolution of disease, and inflammatory markers had normalized. Her treatment had been inactivity, without steroids or other medication.

The patient's condition relapsed six months later and completely settled with prompt steroid medication. Systemically, all was normal and PR3 and MPO markers were negative as were urinalysis and the chest X-ray. Whilst a firm diagnosis could not be made, a granulomatous sinonasal disorder was confirmed by histology.

In discussion, Miss Chevretton suggested isolated relapsing polychondritis or a vasculitis in prodromal phase as likely diagnoses, and Professor Michaels reiterated the fact that midline granuloma is a non-existent condition.

\section{Swelling of the fronto-ethmoid region}

H K Khan, G Dhanasekar, J Mathews From the New Cross Hospital, Wolverhampton, UK

A 59-year-old gentleman presented with a three-month history of a swelling above the right eye. Examination revealed a smooth swelling above the medial canthus of the right eye. An ENT examination, including endoscopy and neck examination, was unremarkable. Clinical examination, including a computed tomography scan, suggested a diagnosis of fronto-ethmoid mucocele. The patient presented a week later with increasing pain and redness. He did not respond to treatment with intravenous antibiotics. Surgical exploration revealed a fleshy mass in the frontal sinus, the histopathology of which was suggestive of spindle cell carcinoma. Following a multidisciplinary team meeting, the patient underwent a radical excision of the tumour, with orbital exenteration and repair.

In discussion, Professor Michaels pointed out that histological diagnosis is challenging and requires immunohistochemistry to differentiate this tumour from a sarcoma. Invariably, a second opinion from a regional experienced pathologist should be sought (in our case, a second histopathological opinion was kindly given by Professor A Malcolm from Shrewsbury). Discussion of such cases at multidisciplinary team meetings is mandatory to enable appropriate management, due to the rarity of this tumour.

A frontal sinus neoplasm can mimic a frontal mucocele at presentation. Computed tomography scans may appear to present benign pathology and can be misleading. A high index of suspicion and early management of these benign-looking lesions are necessary to avoid complications and missed diagnoses.

\section{Teratocarcinosarcoma of the ethmoid sinus}

A Majithia, L Alarcon*, T Tatla, A Sandison*, P Clarke From the Departments of Otorhinolaryngology and *Pathology, Charing Cross Hospital, London, UK

Teratocarcinosarcoma of the nasal cavity and paranasal sinuses is an extremely rare and aggressive tumour. It was first described in 1984 and there have been less than 50 cases reported in the worldwide literature. Histologically, it is composed of a malignant epithelial element and two or more malignant mesenchymal components, with varying degrees of maturity and histological atypia. The variegated nature of the histology and the rarity of the condition make the diagnosis difficult, especially in the presence of small biopsies. Treatment is by surgery and radiotherapy but the prognosis remains poor, with a 60 per cent mortality by three years.

We reported the case of a 73-year-old lady who presented with epistaxis and a rapidly enlarging left nasal mass. Biopsies performed at the referring centre were inconclusive. Imaging showed spread through the cribriform plate into the anterior cranial fossa, and displacement of the orbit without involvement of the medial rectus. The patient underwent craniofacial resection and postoperative radiotherapy. She had persistent, severe left eye pain resistant to analgesics, which was attributed to reflex sympathetic dystrophy. Unfortunately, five months postradiotherapy, she developed a tumour recurrence in her neck. This was treated with orbital exenteration and neck dissection followed by radiotherapy to the neck.

Discussion emphasized the histological variance of tumours within the nasal cavity and the need for both appropriate sampling of specimens and specialist histological review.

\section{Extra-corporeal septorhinoplasty: a surgical technique to straighten gross deviation of the anterior nasal septum}

S A B Ratnayake, S Silva

From St John's Hospital, Mid Essex Hospital Services NHS Trust, Chelmsford, UK

Conventionally, deviation of the nasal septum is managed surgically with sub-mucous resection or septoplasty in the majority of otolaryngology units in the UK. However, gross deviation is resilient to correction and often needs revision surgery. This is due to the inherent elasticity of the cartilage and the tension produced by the unified action of the cartilaginous and bony structures of the nose. We presented extra-corporeal septorhinoplasty (ECS) as a technique to circumvent this problem.

This technique was illustrated with the case of a 40-year-old woman who presented with a long-standing nasal obstruction, without previous trauma or surgery. Examination revealed an S-shaped deformity of the anterior nasal septum and a columella dislocated to the left. The salient points of the ECS technique were presented in three stages (removal, reshaping and replanting of the nasal septum). Fixation of the septum during replanting was emphasized. Three-months after the 
operation, our patient remained symptom-free, with no complications.

In discussion, Mr Roberts (consultant ENT and facial plastics surgeon) recommended using a sterile, absorbable, paper-thin PDS mesh to splint the septum, in addition to the fixation we described in the replanting stage. We believe this technique merits wider consideration by rhinologists for the treatment of gross deviation of the anterior nasal septum.

\section{Management of life-threatening epistaxis; how far can we justifiably go?}

P Ajulo, F Vaz, R Simo, E Chevretton

From Guy's and St Thomas' Hospital, London, UK

We presented the case of a 61-year-old Afro-Caribbean man with recurrent, life-threatening epistaxis. He had been treated in 1987 with radical radiotherapy for undifferentiated nasopharyngeal carcinoma. Thirteen years later, he received re-irradiation for a second primary in the right ethmoid, resulting in osteoradionecrosis of the skull base.

Our patient presented initially with minor, self-limiting epistaxis, progressing to recurrent episodes of torrential epistaxis requiring examination of the nose under general anaesthesia and nasal packing several times. Over the course of two and a half weeks, he had bilateral maxillary artery embolization, right external carotid artery ligation, right anterior ethmoidal artery ligation, right medial maxillectomy, a left external carotid artery ligation and, finally, embolization of the right internal carotid artery. This successfully treated his epistaxis but caused a dense left hemiplegia. The patient was rehabilitated and discharged home nine weeks later, ambulating.

Re-irradiation for head and neck cancer has well documented complications. A very small number of these patients develop recurrent epistaxis, more than one in four resulting in a fatality. In patients who have received re-irradiation for head and neck cancer and who present with epistaxis, embolization should be considered early on. 